\title{
ERROR ANALYSIS OF SOME FINITE ELEMENT METHODS FOR THE STOKES PROBLEM
}

\author{
ROLF STENBERG
}

\begin{abstract}
We prove the optimal order of convergence for some two-dimensional finite element methods for the Stokes equations. First we consider methods of the Taylor-Hood type: the triangular $P_{3}-P_{2}$ element and the $Q_{k}-$ $Q_{k-1}, k \geq 2$, family of quadrilateral elements. Then we introduce two new low-order methods with piecewise constant approximations for the pressure. The analysis is performed using our macroelement technique, which is reviewed in a slightly altered form.
\end{abstract}

\section{INTRODUCTION}

In this paper we will consider some finite element methods for the Stokes equations: find the velocity $\mathbf{u}$ and the pressure $p$ such that

$$
\begin{aligned}
-\nu \Delta \mathbf{u}+\nabla p=\mathbf{f} & \text { in } \boldsymbol{\Omega}, \\
\operatorname{div} \mathbf{u}=0 & \text { in } \Omega, \\
\mathbf{u}=\mathbf{0} & \text { on } \partial \boldsymbol{\Omega},
\end{aligned}
$$

where $\Omega \subset \mathbf{R}^{d}, d=2,3$, is a bounded polygonal or polyhedral domain, $\mathbf{f}$ is the given body force, and $\nu>0$ is the viscosity.

The usual variational formulation of $(1.1)$ is the following. Find $\mathbf{u} \in H_{0}^{1}(\Omega)^{d}$ and $p \in L_{0}^{2}(\Omega)$ such that

$$
\begin{aligned}
\nu(\nabla \mathbf{u}, \nabla \mathbf{v})-(\operatorname{div} \mathbf{v}, p) & =(\mathbf{f}, \mathbf{v}), & & \mathbf{v} \in H_{0}^{1}(\Omega)^{d}, \\
(\operatorname{div} \mathbf{u}, q) & =0, & & q \in L_{0}^{2}(\Omega),
\end{aligned}
$$

where $(\cdot, \cdot)$ denotes the inner product in $L^{2}(\Omega), L^{2}(\Omega)^{d}$ or $L^{2}(\Omega)^{d \times d}$, and $L_{0}^{2}(\Omega)$ the space

$$
L_{0}^{2}(\Omega)=\left\{p \in L^{2}(\Omega) \mid \int_{\Omega} p d \mathbf{x}=0\right\} .
$$

For $\mathbf{f} \in H^{-1}(\Omega)^{d}$ this problem has a unique solution; cf. [11].

Received August 3, 1988.

1980 Mathematics Subject Classification (1985 Revision). Primary 65N30. 
The mixed method based on (1.2) reads as follows. Find $\mathbf{u}_{h} \in \mathbf{V}_{h} \subset H_{0}^{1}(\Omega)^{d}$ and $p_{h} \in P_{h} \subset L_{0}^{2}(\Omega)$ such that

$$
\begin{aligned}
\nu\left(\nabla \mathbf{u}_{h}, \nabla \mathbf{v}\right)-\left(\operatorname{div} \mathbf{v}, p_{h}\right) & =(\mathbf{f}, \mathbf{v}), & & \mathbf{v} \in \mathbf{V}_{h}, \\
\left(\operatorname{div} \mathbf{u}_{h}, q\right) & =0, & & q \in P_{h} .
\end{aligned}
$$

It is well known that in order to get a working method, the spaces $\mathbf{V}_{h}$ and $P_{h}$ cannot be chosen arbitrarily. The method can be expected to behave well only if the following "inf-sup condition" is satisfied:

$$
\inf _{0 \neq p \in P_{h}} \sup _{\mathbf{0} \neq \mathbf{v} \in \mathbf{v}_{h}} \frac{(\operatorname{div} \mathbf{v}, p)}{\|\mathbf{v}\|_{1}\|p\|_{0}} \geq C>0 .
$$

The following fundamental result is classical.

Proposition 1.1 (Babuška [1, 2], Brezzi [6]). Suppose that the finite element spaces $\mathbf{V}_{h}$ and $P_{h}$ satisfy (1.4). Then the system (1.3) has a unique solution $\left(\mathbf{u}_{h}, p_{h}\right)$ satisfying

$$
\left\|\mathbf{u}-\mathbf{u}_{h}\right\|_{1}+\left\|p-p_{h}\right\|_{0} \leq C\left\{\inf _{\mathbf{v} \in \mathbf{v}_{h}}\|\mathbf{u}-\mathbf{v}\|_{1}+\inf _{q \in P_{h}}\|p-q\|_{0}\right\},
$$

where $(\mathbf{u}, p)$ is the solution to (1.2).

By now, this field of problems is rather well understood; there exist rather general techniques for verifying the inf-sup condition [5, 14] and for the construction of methods satisfying it. As a consequence, the collection of methods which are known to be stable (i.e., satisfying (1.4)) is relatively large; cf., e.g., the recent book by Girault and Raviart [11].

The purpose of this paper is to extend the list of stable methods. In $\S 3$ we consider two families of "Taylor-Hood" type methods, and in the last section we introduce and analyze two new low-order methods. In the analysis we use the macroelement technique, introduced by us in [14], which we recall in $\S 2$ in a slightly more practical form.

The results of this paper are trivially also valid when the same finite element spaces are used for the equations of (nearly) incompressible elasticity.

Some of the results of this paper have also been obtained in [7].

Our notation is standard; cf. [8, 11].

\section{THE MACROELEMENT TECHNIQUe}

For further reference we will here present the technique in a quite general form for both two- and three-dimensional problems, even if all the methods to be analyzed in this paper are two-dimensional. The presentation does not cover all possible cases (such as, e.g., a mixing of triangles and quadrilaterals, prismatic elements, etc.), but the modifications needed for a method not covered are trivial.

Let $\mathscr{C}_{h}$ be a finite element partitioning of $\bar{\Omega}$ into subdomains which are all assumed to be either triangles or convex quadrilaterals when $\Omega \subset \mathbf{R}^{2}$, and 
tetrahedrons or convex hexahedrons when $\Omega \subset \mathbf{R}^{3}$. The partitioning is assumed to satisfy the standard regularity and compatibility condition [8], but we will not assume $\mathscr{C}_{h}$ to be quasi-uniform. Let $\widehat{K}$ denote the reference triangle, tetrahedron, square, or cube, respectively, and for $K \in \mathscr{C}_{h}$ denote by $F_{K}$ the affine, bilinear, or trilinear mapping from $\widehat{K}$ onto $K$. Further, let $\widehat{\mathbf{V}}$ and $\widehat{P}$ be two polynomial spaces defined on $\widehat{K}$. We now assume that $\mathbf{V}_{h}$ and $P_{h}$ are defined as

$$
\mathbf{V}_{h}=\left\{\mathbf{v} \in H_{0}^{1}(\Omega)^{d} \mid \mathbf{v}(\mathbf{x})=\hat{\mathbf{v}}\left(F_{K}^{-1}(\mathbf{x})\right), \hat{\mathbf{v}} \in \hat{\mathbf{v}}, K \in \mathscr{C}_{h}\right\},
$$

and

$$
P_{h}=\left\{p \in L_{0}^{2}(\Omega) \mid p(\mathbf{x})=\hat{p}\left(F_{K}^{-1}(\mathbf{x})\right), \hat{p} \in \widehat{P}, K \in \mathscr{C}_{h}\right\},
$$

or

$$
P_{h}=\left\{p \in C(\Omega) \cap L_{0}^{2}(\Omega) \mid p(\mathbf{x})=\hat{p}\left(F_{K}^{-1}(\mathbf{x})\right), \hat{p} \in \widehat{P}, K \in \mathscr{C}_{h}\right\} .
$$

Further, we make the assumption that $\mathbf{V}_{h}$ contains the piecewise linear, isoparametric bilinear, or isoparametric trilinear functions for the triangular and tetrahedral, quadrilateral, or hexahedral case, respectively. With the choice (2.2a), $P_{h}$ is assumed to contain the piecewise constants, whereas for $(2.2 \mathrm{~b})$ it contains the piecewise linear, isoparametric bilinear, or isoparametric trilinear functions.

A method where the pressure space is defined according to $(2.2 b)$ is usually referred to as a "Taylor-Hood" method.

By a macroelement $M$ we define a connected set of elements of which the intersection of any two is either empty, a vertex, or one edge or face in $\mathbf{R}^{2}$ and $\mathbf{R}^{3}$, respectively. Further, two macroelements $M$ and $\widetilde{M}$ are said to be equivalent if they can be mapped continuously onto each other, or more precisely, if one can define a mapping $G: \widetilde{M} \rightarrow M$ such that:

(i) $G(\widetilde{M})=M$.

(ii) If $\widetilde{M}=\bigcup_{j=1}^{m} \widetilde{K}_{j}$, where $\widetilde{K}_{j}, j=1,2, \ldots, m$, are the elements of $\widetilde{M}$, then $K_{j}=G\left(\widetilde{K}_{j}\right), j=1,2, \ldots, m$, are the elements of $M$.

(iii) $G_{\mid \widetilde{K}_{j}}=F_{K_{j}} \circ F_{\widetilde{K}_{j}}^{-1}, j=1,2, \ldots, m$, where $F_{K_{j}}$ and $F_{\widetilde{K}_{j}}$ are the mappings from the reference element $\widehat{K}$ onto $K_{j}$ and $\widetilde{K}_{j}$, respectively.

For a macroelement $M$ we define the spaces

$$
\mathbf{V}_{0, M}=\left\{\mathbf{v} \in H_{0}^{1}(M)^{d} \mid \mathbf{v}(\mathbf{x})=\hat{\mathbf{v}}\left(F_{K}^{-1}(\mathbf{x})\right), \hat{\mathbf{v}} \in \hat{\mathbf{v}}, \mathbf{x} \in K, K \subset M\right\}
$$

and

$$
P_{M}=\left\{p \in L^{2}(M) \mid p(\mathbf{x})=\hat{p}\left(F_{K}^{-1}(\mathbf{x})\right), \hat{p} \in \hat{P}, \mathbf{x} \in K, K \subset M\right\},
$$

or

$$
P_{M}=\left\{p \in C(M) \mid p(\mathbf{x})=\hat{p}\left(F_{K}^{-1}(\mathbf{x})\right), \hat{p} \in \widehat{P}, \mathbf{x} \in K, K \subset M\right\},
$$

depending on which of the possibilities $(2.2 \mathrm{a}, \mathrm{b})$ is chosen. Further, we denote

$$
N_{M}=\left\{p \in P_{M} \mid(\operatorname{div} \mathbf{v}, p)_{M}=0, \mathbf{v} \in \mathbf{V}_{0, M}\right\} .
$$


The collection of edges or faces, of the elements of $\mathscr{C}_{h}$, in the interior of $\Omega$ is denoted by $\Gamma_{h}$.

The macroelement technique is given by the following

Theorem 2.1. Suppose that there is a fixed set of equivalence classes $\mathscr{E}_{i}, i=$ $1,2, \ldots, q$, of macroelements, a positive integer $L$, and a macroelement partitioning $\mathscr{M}_{h}$ such that:

(M1) For each $M \in \mathscr{E}_{i}, i=1,2, \ldots, q$, the space $N_{M}$ is one-dimensional, consisting of functions that are constant on $M$.

(M2) Each $M \in \mathscr{M}_{h}$ belongs to one of the classes $\mathscr{E}_{i}, i=1,2, \ldots, q$.

(M3) Each $K \in \mathscr{C}_{h}$ is contained in at least one and not more than $L$ macroelements of $\mathscr{M}_{h}$.

(M4) Each $T \in \Gamma_{h}$ is contained in the interior of at least one and not more than $L$ macroelements of $\mathscr{M}_{h}$.

Then the stability inequality (1.4) is valid.

Proof. The proof will consist of a modification of some arguments given in [12, 14, 16].

We will need the following norm defined in $P_{h}$ :

$$
\|p\|_{h}^{2}=\sum_{K \in \mathscr{E}_{h}} h_{K}^{2}\|\nabla p\|_{0, K}^{2}+\sum_{T \in \Gamma_{h}} h_{T} \int_{T}|[p]|^{2} d s,
$$

where $([p])_{\mid T}$ denotes the jump in $p$ along the interelement boundary $T$ and $h_{T}$ stands for the length or area of $T$. In a macroelement $M$ we similarly define

$$
|p|_{M}^{2}=\sum_{K \subset M} h_{K}^{2}\|\nabla p\|_{0, K}^{2}+\sum_{T \in \Gamma_{M}} h_{T} \int_{T}|[p]|^{2} d s,
$$

where $\Gamma_{M}$ denotes the interelement boundaries in the interior of $M$.

Now, since we assume that

-there is only a fixed number of different types of macroelements,

-all the elements in $\mathscr{C}_{h}$ are regular, and

-the condition (M1) is satisfied,

one can prove (see [14, Lemma 3.1]) that there is a constant $C$ independent of $\mathscr{M}_{h}$ such that

$$
\inf _{0 \neq p \in P_{M}} \sup _{\mathbf{0} \neq \mathbf{v} \in \mathbf{v}_{0 . M}} \frac{(\operatorname{div} \mathbf{v}, p)_{M}}{|\mathbf{v}|_{1, M}|p|_{M}} \geq C>0,
$$

or alternatively stated: For a given $p \in P_{h}$ and $M \in \mathscr{M}_{h}$ there is a $\mathbf{v}_{M} \in \mathbf{V}_{h}$, with $\mathbf{v}_{M}=\mathbf{0}$ in $\Omega \backslash M$, such that

$$
\left(\operatorname{div} \mathbf{v}_{M}, p\right)_{M} \geq C|p|_{M}^{2}
$$

and

$$
\left|\mathbf{v}_{M}\right|_{1, M} \leq|p|_{M} .
$$


We now define $\mathbf{v} \in \mathbf{V}_{h}$ through $\mathbf{v}=\sum_{M \in \mathscr{M}_{h}} \mathbf{v}_{M}$. This gives

$$
(\operatorname{div} \mathbf{v}, p)=\sum_{M \in \mathscr{M}_{h}}\left(\operatorname{div} \mathbf{v}_{M}, p\right)_{M} \geq C \sum_{M \in \mathscr{M}_{h}}|p|_{M}^{2} \geq C\|p\|_{h}^{2}
$$

and

$$
\|\mathbf{v}\|_{1} \leq C|\mathbf{v}|_{1} \leq C \sum_{M \in \mathscr{K}_{h}}\left|\mathbf{v}_{M}\right|_{1, M} \leq C \sum_{M \in \mathscr{M}_{h}}|p|_{M} \leq C L\|p\|_{h},
$$

i.e., there is a constant $C_{1}>0$ such that

$$
\sup _{\mathbf{0} \neq \mathbf{v} \in \mathbf{V}_{h}} \frac{(\operatorname{div} \mathbf{v}, p)}{\|\mathbf{v}\|_{1}} \geq C_{1}\|p\|_{h} .
$$

Next, let us use an argument due to Verfürth [16, Proposition 3.3] for showing that (2.9) implies the corresponding condition with the $L^{2}$-norm for the pressure, i.e., the inf-sup condition (1.4). We will show that the argument can be used also when $P_{h}$ consists of discontinuous functions and that the quasiuniformity assumption of [16] can be avoided.

Now, for every $p \in P_{h}$ there is a $\mathbf{w} \in H_{0}^{1}(\Omega)^{d}$ such that

$$
(\operatorname{div} \mathbf{w}, p) \geq C_{2}\|p\|_{0}^{2}
$$

and

$$
\|\mathbf{w}\|_{1} \leq\|p\|_{0} .
$$

Further, one can show (cf. [9; 3, Lemma 3; 11, pp. 109-111]) that there is an interpolant $\tilde{\mathbf{w}} \in \mathbf{V}_{h}$ to $\mathbf{w}$ such that

$$
\left(\sum_{K \in \mathscr{C}_{h}} h_{K}^{-2}\|\mathbf{w}-\tilde{\mathbf{w}}\|_{0, K}^{2}+\sum_{T \in \Gamma_{h}} h_{T}^{-1} \int_{T}|\mathbf{w}-\tilde{\mathbf{w}}|^{2} d s\right)^{1 / 2} \leq C_{3}|\mathbf{w}|_{1}
$$

and

$$
\|\tilde{\mathbf{w}}\|_{1} \leq C_{4}\|\mathbf{w}\|_{1}
$$

Using (2.10), (2.12), and (2.11), we now get

$$
\begin{aligned}
(\operatorname{div} \tilde{\mathbf{w}}, p)=(\operatorname{div}(\tilde{\mathbf{w}}-\mathbf{w}), p)+(\operatorname{div} \mathbf{w}, p) \\
\quad \geq(\operatorname{div}(\tilde{\mathbf{w}}-\mathbf{w}), p)+C_{2}\|p\|_{0}^{2} \\
\quad=\sum_{K \in \mathscr{C}_{h}}(\mathbf{w}-\tilde{\mathbf{w}}, \nabla p)_{K}+\sum_{T \in \Gamma_{h}} \int_{T}((\tilde{\mathbf{w}}-\mathbf{w}) \cdot \mathbf{n})([p]) d s+C_{2}\|p\|_{0}^{2} \\
\quad \geq-\left(\sum_{K \in \mathscr{C}_{h}} h_{K}^{-2}\|\mathbf{w}-\tilde{\mathbf{w}}\|_{0, K}^{2}+\sum_{T \in \Gamma_{h}} h_{T}^{-1} \int_{T}|\mathbf{w}-\tilde{\mathbf{w}}|^{2} d s\right)^{1 / 2} \cdot\|p\|_{h}+C_{2}\|p\|_{0}^{2} \\
\quad \geq-C_{3}|\mathbf{w}|_{1}\|p\|_{h}+C_{2}\|p\|_{0}^{2} \\
\geq-C_{3}\|p\|_{0}\|p\|_{h}+C_{2}\|p\|_{0}^{2} \\
=\|p\|_{0}^{2} \cdot\left(C_{2}-C_{3} \frac{\|p\|_{h}}{\|p\|_{0}}\right),
\end{aligned}
$$


which combined with (2.11) and (2.13) gives

$$
\sup _{\mathbf{0} \neq \mathbf{v} \in \mathbf{v}_{h}} \frac{(\operatorname{div} \mathbf{v}, p)}{\|\mathbf{v}\|_{1}} \geq\|p\|_{0} \cdot\left(C_{5}-C_{6} \frac{\|p\|_{h}}{\|p\|_{0}}\right) \text {. }
$$

On the other hand, (2.9) can be written as

$$
\sup _{\mathbf{0} \neq \mathbf{v} \in \mathbf{v}_{h}} \frac{(\operatorname{div} \mathbf{v}, p)}{\|\mathbf{v}\|_{1}} \geq C_{1}\|p\|_{h}=\|p\|_{0} \cdot C_{1} \frac{\|p\|_{h}}{\|p\|_{0}} .
$$

Hence, combining (2.14) and (2.15), we get

$$
\inf _{0 \neq p \in P_{h}} \sup _{\mathbf{0} \neq \mathbf{v} \in \mathbf{v}_{h}} \frac{(\operatorname{div} \mathbf{v}, p)}{\|\mathbf{v}\|_{1}\|p\|_{0}} \geq \min _{t>0} \max \left\{C_{1} t, C_{5}-C_{6} t\right\}=\frac{C_{1} C_{5}}{C_{1}+C_{6}} .
$$

Let us close this section by giving some remarks on the difference between the formulation of the macroelement technique given in [14] and that of this paper. In [14], the macroelement partitioning $\mathscr{M}_{h}$ was chosen so that each $K \in \mathscr{C}_{h}$ was a subset of exactly one $M \in \mathscr{M}_{h}$. Then the condition (M1) ensures that we can stabilize all pressure components except those which consist of constants on each macroelement. To stabilize the piecewise constant pressure components, we clearly need velocity degrees of freedom in the interior of the boundaries between the macroelements. More precisely, we included the condition that for any two neighboring macroelements $M_{1}, M_{2}$ of $\mathscr{M}_{h}$ with $\int_{M_{1} \cap M_{2}} d s \neq 0$, there is a $\mathbf{v} \in \mathbf{V}_{h}$ such that

$$
\text { supp v } \subset M_{1} \cup M_{2} \text { and } \int_{M_{1} \cap M_{2}} \mathbf{v} \cdot \mathbf{n} d s \neq 0 .
$$

The disadvantage of this choice is that in order to find a macroelement partitioning of nonoverlapping macroelements with this property, it is often necessary to use many different kinds of macroelements or macroelements that are big. In these cases one could claim that the asymptotic stability inequality so obtained is not valid for most values of the mesh parameter used in practice. In many cases it is not even evident how such a macroelement partitioning should be obtained. A good example showing these problems is the tetrahedral Taylor-Hood method. In [15] we analyzed this method with the technique of this paper. The present technique also shows more clearly that the condition of the space $N_{M}$ to consist of constants is really the only one that has to be verified.

We now use the above theorem for the analysis of some mixed methods not earlier analyzed in the literature.

\section{TWO FAMILIES OF METHODS}

Let $\Omega \subset \mathbf{R}^{2}$, and let $\mathscr{C}_{h}$ be a partitioning, the elements of which all are assumed to be either triangles or quadrilaterals. For the index $k \geq 2$ we define the families

$$
\begin{aligned}
& \mathbf{v}_{h}=\left\{\mathbf{v} \in H_{0}^{1}(\Omega)^{2} \mid \mathbf{v}_{\mid K} \in R_{k}(K)^{2}, K \in \mathscr{C}_{h}\right\}, \\
& P_{h}=\left\{p \in L_{0}^{2}(\Omega) \cap C(\Omega) \mid p_{\mid K} \in R_{k-1}(K), K \in \mathscr{C}_{h}\right\},
\end{aligned}
$$


where $R_{m}(K)=P_{m}(K)$ when $K$ is a triangle, and $R_{m}(K)=Q_{m}(K)$ when $K$ is a quadrilateral. $P_{m}(K)$ and $Q_{m}(K)$ are the usual polynomial spaces on $K$; cf. [8].

For these families we have the following optimal error estimates.

Theorem 3.1. For the solution $\left(\mathbf{u}_{h}, p_{h}\right)$ of (1.3) with the method (3.1) we have

$$
\left\|\mathbf{u}-\mathbf{u}_{h}\right\|_{1}+\left\|p-p_{h}\right\|_{0} \leq C h^{k}\left(|\mathbf{u}|_{k+1}+|p|_{k}\right) .
$$

For a convex domain $\Omega$ we additionally have

$$
\left\|\mathbf{u}-\mathbf{u}_{h}\right\|_{0} \leq C h^{k+1}\left(|\mathbf{u}|_{k+1}+|p|_{k}\right) .
$$

For the triangular case, the verification of the above estimates consists merely of filling in some gaps; for $k \geq 4$ the result is covered by the analysis in [13] and for $k=2$ the result is well known [4, 16], but under the restrictions that no $K \in \mathscr{C}_{h}$ has two edges on the boundary $\partial \Omega$ and that $\mathscr{C}_{h}$ is quasi-uniform. We will prove stability for the case $k=3$. Exactly the same line of reasoning applies in the case $k=2$, and hence the above-mentioned restrictions on the mesh are unnecessary.

Remark. In [15] we analyzed the corresponding three-dimensional tetrahedral method for $k=2$. There, the restriction on the mesh was that each tetrahedron in $\mathscr{C}_{h}$ has at least one vertex in the interior of $\Omega$. An inspection shows, however, that this restriction cannot be dropped.

Lemma 3.1. Let $M$ be a macroelement consisting of three triangles, and define

$$
\begin{aligned}
\mathbf{v}_{0, M} & =\left\{\mathbf{v} \in H_{0}^{1}(M)^{2} \mid \mathbf{v}_{\mid K} \in P_{3}(K)^{2}, K \subset M\right\}, \\
P_{M} & =\left\{p \in C(M) \mid p_{\mid K} \in P_{2}(K), K \subset M\right\} .
\end{aligned}
$$

Then the corresponding nullspace $N_{M}$ is one-dimensional, consisting of functions that are constant on $M$.

Proof. Let $M=K_{1} \cup K_{2} \cup K_{3}$ be as in Figure 1, and let $\mathbf{t}_{12}, \mathbf{t}_{23}$ and $\mathbf{n}_{12}, \mathbf{n}_{23}$ be the tangents and normals, respectively, to the common edges. Let us first consider $K_{1} \cup K_{2}$ with the "local numbering" of the vertices as in the figure. For $p \in P_{M}$ we have $\nabla p_{\mid K_{j}} \in P_{1}\left(K_{j}\right)^{2}, j=1,2$, and the component $\nabla p \cdot \mathbf{t}_{12}$ is continuous in the whole of $K_{1} \cup K_{2}$. Hence, we can write

$$
\left(\nabla p \cdot \mathbf{t}_{12}\right)_{\mid K_{j}}=\sum_{i=1}^{3} \alpha_{i j} \lambda_{i j}, \quad j=1,2,
$$

where $\lambda_{i j}, i=1,2,3$, are the barycentric coordinates of $K_{j}, j=1,2$. Since $\nabla p \cdot \mathbf{t}_{12}$ is continuous in $K_{1} \cup K_{2}$, we have $\alpha_{i 1}=\alpha_{i 2}$ for $i=1,2$. Now, choose $\mathbf{u} \in \mathbf{V}_{0, K_{1} \cup K_{2}}$ (i.e., $\mathbf{u}=\mathbf{0}$ in $K_{3}$ ) such that $\mathbf{u} \cdot \mathbf{n}_{12}=0$ in $K_{1} \cup K_{2}$ and

$$
\left(\mathbf{u} \cdot \mathbf{t}_{12}\right)_{\mid K_{j}}=\lambda_{1 j} \lambda_{2 j}\left(3 \lambda_{1 j}-1\right), \quad j=1,2 .
$$




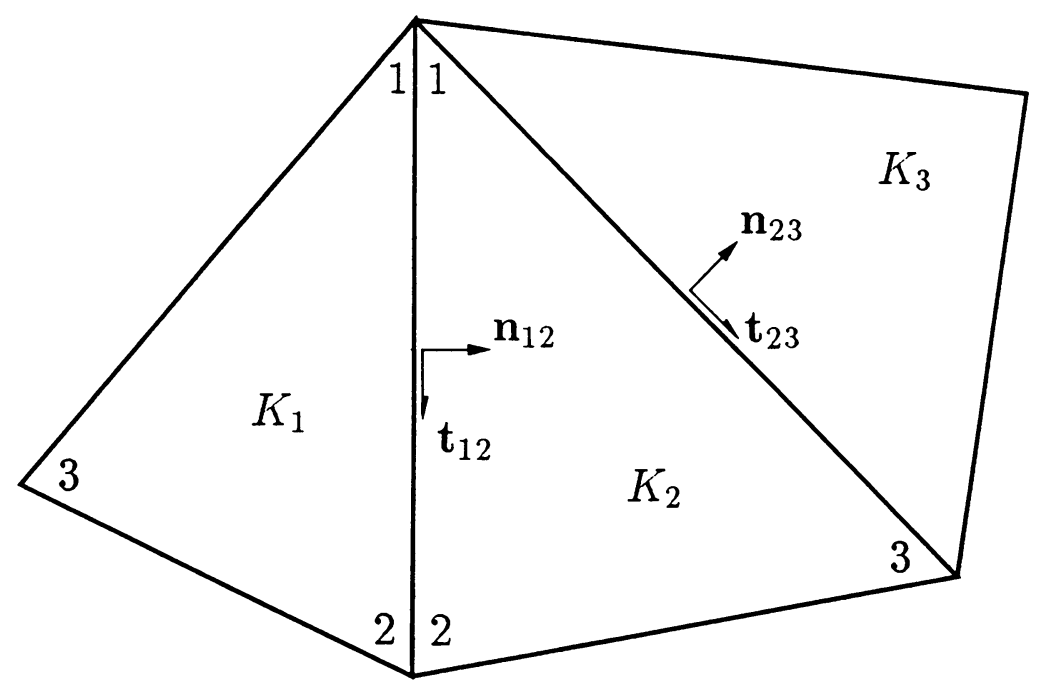

Figure 1

Then we have

$$
\begin{aligned}
(\operatorname{div} \mathbf{u}, p)_{M} & =-(\mathbf{u}, \nabla p)_{M}=-\sum_{j=1}^{2} \int_{K_{j}}\left(\mathbf{u} \cdot \mathbf{t}_{12}\right)\left(\nabla p \cdot \mathbf{t}_{12}\right) d \mathbf{x} \\
& =-\sum_{j=1}^{2} \sum_{i=1}^{3} \alpha_{i j} \int_{K_{j}} \lambda_{1 j} \lambda_{2 j}\left(3 \lambda_{1 j}-1\right) \lambda_{i j} d \mathbf{x} \\
& =-\frac{\operatorname{area}\left(K_{1}\right)}{60} \alpha_{11}-\frac{\operatorname{area}\left(K_{2}\right)}{60} \alpha_{12} \\
& =-\left(\frac{\operatorname{area}\left(K_{1}\right)}{60}+\frac{\operatorname{area}\left(K_{2}\right)}{60}\right) \alpha_{12}
\end{aligned}
$$

and the condition $(\operatorname{div} \mathbf{u}, p)_{M}=0$ implies that $\alpha_{11}=\alpha_{12}=0$. By symmetric reasoning we get $\alpha_{21}=\alpha_{22}=0$. Next we choose $\mathbf{u} \in \mathbf{V}_{0, K_{j}}, j=1,2$, such that $\mathbf{u} \cdot \mathbf{n}_{12}=0$ and

$$
\left(\mathbf{u} \cdot \mathbf{t}_{12}\right)_{\mid K_{j}}=\lambda_{1 j} \lambda_{2 j} \lambda_{3 j}, \quad j=1,2 .
$$

If $p \in N_{M}$ then we have

$$
\begin{aligned}
0 & =(\operatorname{div} \mathbf{u}, p)_{M}=-(\mathbf{u}, \nabla p)_{M} \\
& =-\alpha_{3 j} \int_{K_{j}} \lambda_{1 j} \lambda_{2 j} \lambda_{3 j}^{2} d \mathbf{x}=-\frac{\operatorname{area}\left(K_{j}\right)}{180} \alpha_{3 j}, \quad j=1,2,
\end{aligned}
$$

that is, $\alpha_{3 j}=0, j=1,2$. We have thus shown that

$$
\nabla p \cdot \mathbf{t}_{12}=0 \text { in } K_{1} \cup K_{2} .
$$

The same reasoning gives that

$$
\nabla p \cdot \mathbf{t}_{23}=0 \text { in } K_{2} \cup K_{3},
$$


and hence we have

$$
\nabla p=\mathbf{0} \text { in } K_{2}
$$

i.e., $p$ is a constant in $K_{2}$.

Write next

$$
\left(\nabla p \cdot \mathbf{n}_{12}\right)_{\mid K_{1}}=\sum_{i=1}^{3} \beta_{i} \lambda_{i 1}
$$

and take $\mathbf{u} \in \mathbf{V}_{0, K_{1} \cup K_{2}}$ such that $\mathbf{u} \cdot \mathbf{t}_{12}=0$ and

$$
\left(\mathbf{u} \cdot \mathbf{n}_{12}\right)_{\mid K_{j}}=\lambda_{1 j} \lambda_{2 j}\left(3 \lambda_{1 j}-1\right), \quad j=1,2 .
$$

Now, since $\nabla p_{\mid K_{2}}=\mathbf{0}$, the condition for $N_{M}$ yields

$$
\begin{aligned}
0 & =(\operatorname{div} \mathbf{u}, p)_{M}=-(\mathbf{u}, \nabla p)_{K_{1}} \\
& =-\sum_{i=1}^{3} \beta_{i} \int_{K_{1}} \lambda_{11} \lambda_{21}\left(3 \lambda_{11}-1\right) \lambda_{i 1} d \mathbf{x}=-\frac{\operatorname{area}\left(K_{1}\right)}{60} \beta_{1},
\end{aligned}
$$

i.e., $\beta_{1}=0$, and analogously we get $\beta_{2}=0$. Upon choosing $\mathbf{u} \in \mathbf{V}_{0, K_{1}}$ such that $\mathbf{u} \cdot \mathbf{t}_{12}=0$ and $\left(\mathbf{u} \cdot \mathbf{n}_{12}\right)_{\mid K_{1}}=\lambda_{11} \lambda_{21} \lambda_{31}$, we get

$$
0=(\operatorname{div} \mathbf{u}, p)_{M}=(\operatorname{div} \mathbf{u}, p)_{K_{1}}=-\frac{\operatorname{area}\left(K_{1}\right)}{180} \beta_{3},
$$

i.e., $\beta_{3}=0$ in $K_{1}$. We thus have $\nabla p \cdot \mathbf{n}_{12}=0$ in $K_{1}$, and hence $p$ is a constant in $K_{1}$. Analogously, $p \in N_{M}$ has to be a constant in $K_{3}$.

Since $p \in N_{M}$ is a constant in $K_{i}, i=1,2,3$, and continuous, it is constant in the whole of $M$.

When applying Theorem 2.1, we now use only one class of macroelements, and the partitioning $\mathscr{M}_{h}$ can be obtained by, e.g., taking one macroelement for each element edge in the interior of $\Omega$. Then the assumptions of Theorem 2.1 are valid, and standard approximation theory gives the first estimate of Theorem 3.1. The $L^{2}$-estimate for the velocity follows by the standard AubinNitsche trick.

Let us turn to the quadrilateral family. To our knowledge, only the lowestorder method in this family has earlier been analyzed [4, 14].

Lemma 3.2. Let $M$ be a macroelement consisting of two quadrilaterals, and define

$$
\begin{gathered}
\mathbf{V}_{0, M}=\left\{\mathbf{v} \in H_{0}^{1}(M)^{2} \mid \mathbf{v}_{\mid K} \in Q_{k}(K)^{2}, K \subset M\right\} \\
P_{M}=\left\{p \in C(M) \mid p_{\mid K} \in Q_{k-1}(K), K \subset M\right\} .
\end{gathered}
$$

Then the corresponding nullspace $N_{M}$ is one-dimensional, consisting of functions that are constant on $M$.

Proof. Let $M=K_{1} \cup K_{2}$ and $\widehat{M}=\widehat{K}_{1} \cup \widehat{K}_{2}$ be the macroelement and the reference macroelement, respectively, and denote by $F=\left(F_{1}, F_{2}\right)$ the continuous 


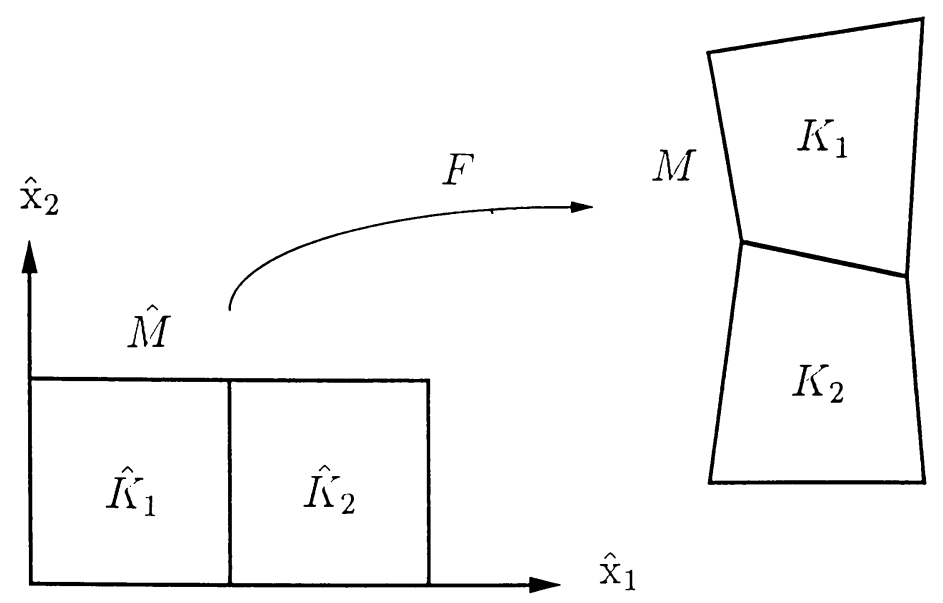

FIGURE 2

piecewise bilinear mapping from $\widehat{M}$ onto $M$ as in Figure 2. For $\mathbf{u} \in \mathbf{V}_{0, M}$ and $p \in N_{M}$, we now have

$$
\begin{aligned}
0 & =-(\operatorname{div} \mathbf{u}, p)_{M}=(\mathbf{u}, \nabla p)_{M} \\
& =\sum_{i=1}^{2} \int_{\widehat{K}_{i}} \hat{\mathbf{u}}(\hat{\mathbf{x}})^{T} J_{F}^{-T}(\hat{\mathbf{x}}) \nabla \hat{p}(\hat{\mathbf{x}})\left|J_{F}(\hat{\mathbf{x}})\right| d \hat{\mathbf{x}},
\end{aligned}
$$

where $J_{F}$ is the Jacobian matrix of $F, J_{F}^{-T}$ is the transpose of $J_{F}^{-1}$, and $\left|J_{F}\right|$ is the determinant of $J_{F}$. Above, $\hat{\mathbf{u}}(\hat{\mathbf{x}})$ and $\nabla \hat{p}(\hat{\mathbf{x}})$ are considered to be column vectors. We now have

$$
\left|J_{F}(\hat{\mathbf{x}})\right| J_{F}^{-T}(\hat{\mathbf{x}})=\left(\begin{array}{cc}
\partial_{2} F_{2}(\hat{\mathbf{x}}) & -\partial_{1} F_{2}(\hat{\mathbf{x}}) \\
-\partial_{2} F_{1}(\hat{\mathbf{x}}) & \partial_{1} F_{1}(\hat{\mathbf{x}})
\end{array}\right) .
$$

Since $F_{1}$ and $F_{2}$ are bilinear, this shows that

$$
\left[\left|J_{F}(\hat{\mathbf{x}})\right| J_{F}^{-T}(\hat{\mathbf{x}}) \nabla \hat{p}(\hat{\mathbf{x}})\right]_{\mid \widehat{K}_{i}} \in Q_{k-1}\left(\widehat{K}_{i}\right)^{2}, \quad i=1,2,
$$

and hence

$$
\left[\hat{\mathbf{u}}(\hat{\mathbf{x}})^{T} J_{F}^{-T}(\hat{\mathbf{x}}) \nabla \hat{p}(\hat{\mathbf{x}})\left|J_{F}(\hat{\mathbf{x}})\right|\right]_{\mid \widehat{K}_{i}} \in Q_{2 k-1}\left(\widehat{K}_{i}\right), \quad i=1,2 .
$$

This means that the integrals

$$
\int_{\widehat{K}_{i}} \hat{\mathbf{u}}(\hat{\mathbf{x}})^{T} J_{F}^{-T}(\hat{\mathbf{x}}) \nabla \hat{p}(\hat{\mathbf{x}})\left|J_{F}(\hat{\mathbf{x}})\right| d \hat{\mathbf{x}}, \quad i=1,2,
$$

are exactly evaluated by the composite $(k+1)$-point Gauss-Lobatto formula.

On the other hand, we can for the nodal degrees of freedom of $\mathbf{u}$ choose the values at the $(k+1)^{2}$ Gauss-Lobatto points in $\widehat{K}_{i}, i=1,2$.

Denote by $0=a_{0}, a_{1}, \ldots, a_{k}=1$ the Gauss-Lobatto points in the unit interval, so that the Gauss-Lobatto points in the two squares are given by

$$
\hat{\mathbf{x}}_{i j}^{1}=\left(a_{i}, a_{j}\right), \quad i, j=0,1, \ldots, k,
$$


and

$$
\hat{\mathbf{x}}_{i j}^{2}=\left(a_{i}+1, a_{j}\right), \quad i, j=0,1, \ldots, k .
$$

If we now choose $\hat{\mathbf{u}}$ such that its only nonvanishing degree of freedom is successively one of the components at the Gauss-Lobatto points in the interior of $\widehat{K}_{1}$ and $\widehat{K}_{2}$, we get

$$
J_{F}^{-T}\left(\hat{\mathbf{x}}_{i j}^{m}\right) \nabla \hat{p}\left(\hat{\mathbf{x}}_{i j}^{m}\right)\left|J_{F}\left(\hat{\mathbf{x}}_{i j}^{m}\right)\right|=\mathbf{0}, \quad i, j=1,2, \ldots, k-1, m=1,2 .
$$

Since $\left|J_{F}(\hat{\mathbf{x}})\right| \neq 0$ for all $\hat{\mathbf{x}}$, this shows that

$$
\nabla \hat{p}\left(\hat{\mathbf{x}}_{i j}^{m}\right)=\mathbf{0}, \quad i, j=1,2, \ldots, k-1, \quad m=1,2 .
$$

Next, by choosing $\hat{\mathbf{u}}$ so that the only nonvanishing degree of freedom is one of the components at $\hat{\mathbf{x}}_{k j}^{1}=\hat{\mathbf{x}}_{0 j}^{2}, j=1,2, \ldots, k-1$, we get the condition

$$
\begin{array}{r}
J_{F}^{-T}\left(\hat{\mathbf{x}}_{k j}^{1}\right) \nabla \hat{p}\left(\hat{\mathbf{x}}_{k j}^{1}\right)\left|J_{F}\left(\hat{\mathbf{x}}_{k j}^{1}\right)\right|+J_{F}^{-T}\left(\hat{\mathbf{x}}_{0 j}^{2}\right) \nabla \hat{p}\left(\hat{\mathbf{x}}_{0 j}^{2}\right)\left|J_{F}\left(\hat{\mathbf{x}}_{0 j}^{2}\right)\right|=\mathbf{0}, \\
j=1,2, \ldots, k-1 .
\end{array}
$$

Here, the values at the points $\hat{\mathbf{x}}_{k j}^{1}, j=1,2, \ldots, k-1$, are the limits

$$
\lim _{\substack{\hat{\mathbf{x}} \rightarrow \hat{\mathbf{x}}_{k j}^{1} \\ \hat{\mathbf{x}} \in \widehat{K}_{1}}} J_{F}^{-T}(\hat{\mathbf{x}}) \nabla \hat{p}(\hat{\mathbf{x}})\left|J_{F}(\hat{\mathbf{x}})\right|
$$

and analogously for $\hat{\mathbf{x}}_{0 j}^{2}, j=1,2, \ldots, k-1$. At the common edge $\widehat{K}_{1} \cap \widehat{K}_{2}$, $\partial_{2} \hat{p}$ is continuous, and an inspection shows that this is also the case with $\partial_{2} F_{i}$, $i=1,2$. Hence, using (3.2), the condition (3.4) reduces to

$$
\left(\begin{array}{cc}
\partial_{2} F_{2}\left(\hat{\mathbf{x}}_{k j}^{1}\right) & -\partial_{1} F_{2}\left(\hat{\mathbf{x}}_{k j}^{1}\right)-\partial_{1} F_{2}\left(\hat{\mathbf{x}}_{0 j}^{2}\right) \\
-\partial_{2} F_{1}\left(\hat{\mathbf{x}}_{k j}^{1}\right) & \partial_{1} F_{1}\left(\hat{\mathbf{x}}_{k j}^{1}\right)+\partial_{1} F_{1}\left(\hat{\mathbf{x}}_{0 j}^{2}\right)
\end{array}\right)\left(\begin{array}{c}
\partial_{1} \hat{p}\left(\hat{\mathbf{x}}_{k j}^{1}\right)+\partial_{1} \hat{p}\left(\hat{\mathbf{x}}_{0 j}^{2}\right) \\
\partial_{2} \hat{p}\left(\hat{\mathbf{x}}_{k j}^{1}\right)
\end{array}\right)=\mathbf{0} .
$$

Further, at a point $\hat{\mathbf{x}} \in \widehat{K}_{1} \cup \widehat{K}_{2}$, the vectors

$$
\left(\partial_{2} F_{2}(\hat{\mathbf{x}}),-\partial_{2} F_{1}(\hat{\mathbf{x}})\right)^{T} \text { and }\left(-\partial_{1} F_{2}(\hat{\mathbf{x}}), \partial_{1} F_{1}(\hat{\mathbf{x}})\right)^{T}
$$

are the normals to $\left(\partial_{2} F_{1}(\hat{\mathbf{x}}), \partial_{2} F_{2}(\hat{\mathbf{x}})\right)^{T}$ and $\left(\partial_{1} F_{1}(\hat{\mathbf{x}}), \partial_{1} F_{2}(\hat{\mathbf{x}})\right)^{T}$, respectively. Hence, since $K_{1}$ and $K_{2}$ are regular, the determinant of the coefficient matrix in (3.5) is nonvanishing, and we get

$$
\partial_{1} \hat{p}\left(\hat{\mathbf{x}}_{k j}^{1}\right)+\partial_{1} \hat{p}\left(\hat{\mathbf{x}}_{0 j}^{2}\right)=0, \quad j=1,2, \ldots, k-1,
$$

and

$$
\partial_{2} \hat{p}\left(\hat{\mathbf{x}}_{k j}^{1}\right)=0, \quad j=1,2, \ldots, k-1 .
$$

Now (3.3) and (3.6) shows that $\partial_{2} \hat{p}$ vanishes at the points $\hat{\mathbf{x}}_{i j}^{1}, i=1,2, \ldots$, $k, j=1,2, \ldots, k-1$, and $\hat{\mathbf{x}}_{i j}^{2}, i=0,1, \ldots, k-1, j=1,2, \ldots$, $k-1$. Since $\partial_{2} \hat{p}_{\mid \widehat{K}_{i}} \in Q_{k-1, k-2}\left(\widehat{K}_{i}\right), i=1,2$ (i.e., $\partial_{2} \hat{p}_{\mid \widehat{K}_{i}}$ is of the form 
$\left.\sum_{i=0}^{k-1} \sum_{j=0}^{k-2} a_{i j} \hat{x}_{1}^{i} \hat{x}_{2}^{j}, \hat{\mathbf{x}}=\left(\hat{x}_{1}, \hat{x}_{2}\right)\right)$, and the above set of points are unisolvent in $Q_{k-1, k-2}\left(\widehat{K}_{i}\right), i=1,2$, we conclude that

$$
\partial_{2} \hat{p}=0 \text { in } \widehat{K}_{1} \cup \widehat{K}_{2} \text {. }
$$

As a consequence, $\hat{p}_{\mid \widehat{K}_{i}}, i=1,2$, is a polynomial of degree $k-1$ in the $\hat{x}_{1}$-variable alone. This mean that (3.3) also implies

$$
\partial_{1} \hat{p}=0 \text { in } \widehat{K}_{1} \cup \widehat{K}_{2} \text {. }
$$

Owing to the continuity of $\hat{p},(3.7)$ and (3.8) imply that $\hat{p}$ is a constant in $\widehat{M}$. The assertion is thus proved.

In the macroelement partitioning we now again choose one macroelement for each element edge in the interior of $\Omega$. Hence, Proposition 1.1 and the Aubin-Nitsche trick gives the estimates of Theorem 3.1.

\section{TWO LOW-ORDER METHODS}

Let again $\Omega \subset \mathbf{R}^{2}$, and let $\mathscr{C}_{h}$ be a regular finite element partitioning of $\bar{\Omega}$ into triangles or quadrilaterals. (It will become clear that one also could use a mesh with both triangles and quadrilaterals.) The finite element method is now defined through

$$
P_{h}=\left\{p \in L_{0}^{2}(\Omega) \mid p_{\mid K} \in P_{0}(K), K \in \mathscr{C}_{h}\right\}
$$

and

$$
\mathbf{V}_{h}=\left\{\mathbf{v}=\left(v_{1}, v_{2}\right) \in H_{0}^{1}(\Omega)^{2} \mid v_{1 \mid K} \in R_{1}(K), v_{2 \mid K} \in S(K), K \in \mathscr{C}_{h}\right\},
$$

where $R_{1}(K)=P_{1}(K)$ if $K$ is a triangle, and $R_{1}(K)=Q_{1}(K)$ for a quadrilateral. For triangles we let $S(K)=P_{2}(K)$, and for quadrilaterals we define

$$
S(K)=\left\{g \mid g(\mathbf{x})=\hat{g}\left(F_{K}^{-1}(\mathbf{x})\right), \hat{g} \in Q_{2}^{\prime}(\widehat{K})\right\},
$$

where $F_{K}$ is the bilinear mapping from the reference square $\widehat{K}$ onto $K$ and $Q_{2}^{\prime}(\widehat{K})$ is the space of "reduced biquadratic polynomials" (i.e., the quadratic serendipity element) as defined in, e.g., [8, p. 63]. For a macroelement, we analogously define

$$
\mathbf{V}_{0, M}=\left\{\mathbf{v}=\left(v_{1}, v_{2}\right) \in H_{0}^{1}(M)^{2} \mid v_{1 \mid K} \in R_{1}(K), v_{2 \mid K} \in S(K), K \subset M\right\}
$$

and

$$
P_{M}=\left\{p \in L^{2}(M) \mid p_{\mid K} \in P_{0}(K), K \subset M\right\} .
$$

Let us now verify the following

Lemma 4.1. Let $M$ be a macroelement consisting of elements which all have one common vertex in the interior of $M$. Then $N_{M}$ consists of functions that are constant on $M$.

Proof. Let $K_{i}, i=1,2, \ldots \kappa$, be the elements of $M$, and denote $p_{i}=p_{\mid K_{i}}$, $i=1,2, \ldots, \kappa$. Further, let $\mathbf{x}_{i}$ and $\mathbf{n}_{i}, i=1,2, \ldots, \kappa$, be the midpoints 


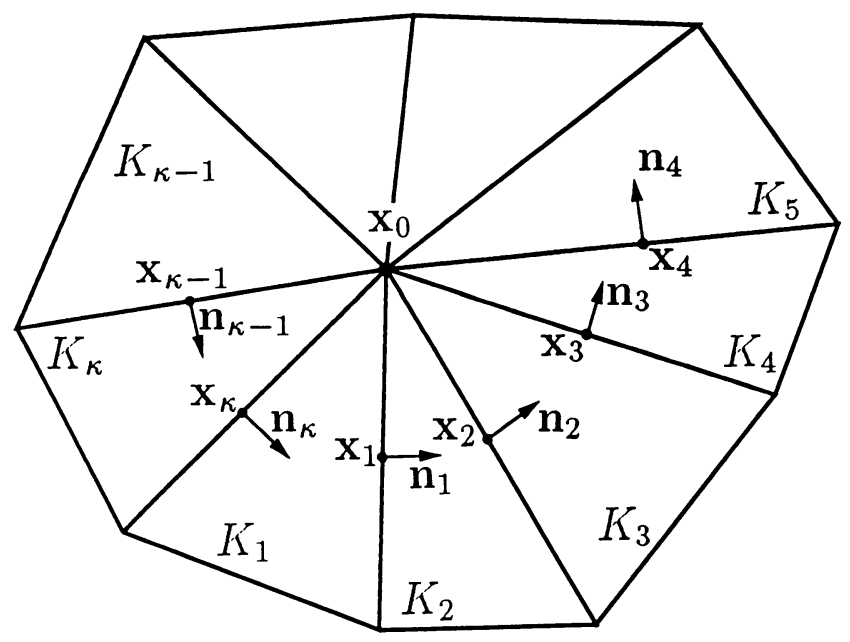

FIGURE 3

and normals, respectively, to the edges in $M$. The common vertex we denote by $\mathbf{x}_{0}$, see Figure 3 for the triangular case.

The natural degrees of freedom for $\mathbf{u} \in \mathbf{V}_{0, M}$ are the values of both components of $\mathbf{u}$ at $\mathbf{x}_{0}$ and the values of $u_{2}$ at $\mathbf{x}_{i}, i=1,2, \ldots, \kappa$. By taking $\mathbf{u} \in \mathbf{V}_{0, M}$ such that the only nonvanishing degree of freedom is $u_{2}\left(\mathbf{x}_{i}\right)$, the condition $(\operatorname{div} \mathbf{u}, p)_{M}=0$ implies that $p_{i}=p_{i+1}\left(\right.$ with $\left.p_{\kappa+1}=p_{0}\right)$ if $\mathbf{n}_{i} \cdot \mathbf{e}_{2} \neq 0$, where $\mathbf{e}_{2}=(0,1)$. Hence, the space $N_{M}$ can be at most two-dimensional, and this happens only if two of the edges are parallel to $e_{2}$. But in this case one chooses $\mathbf{u}$ such that the only nonzero degree of freedom is $u_{1}\left(\mathbf{x}_{0}\right)$. The condition for $N_{M}$ then forces $p$ to be constant on the whole of $M$.

If we now impose the restriction on $\mathscr{C}_{h}$ that every $K \in \mathscr{C}_{h}$ has at least one vertex in the interior of $\Omega$, then we can easily construct a macroelement partitioning $\mathscr{M}_{h}$ satisfying (M1)-(M4): For each interior vertex of the mesh one takes one macroelement consisting of all the elements which have this vertex in common.

Hence we get the following

Theorem 4.1. Suppose that every $K \in \mathscr{C}_{h}$ has at least one vertex in the interior of $\Omega$. Then we have

$$
\left\|\mathbf{u}-\mathbf{u}_{h}\right\|_{1}+\left\|p-p_{h}\right\|_{0} \leq C h\left(|\mathbf{u}|_{2}+|p|_{1}\right) .
$$

For a convex domain $\Omega$ we additionally have

$$
\left\|\mathbf{u}-\mathbf{u}_{h}\right\|_{0} \leq C h^{2}\left(|\mathbf{u}|_{2}+|p|_{1}\right) \text {. }
$$

Remark. The elements we have presented here are rather similar to two elements by Fortin [10]. In Fortin's methods, piecewise constants are used for the pressure, whereas the basic velocity spaces of linear/bilinear functions are augmented with quadratic functions which have as degrees of freedom the normal 
components of the velocity at the midpoints of the element edges. Hence, our methods are simpler to implement. The calculation of the element matrices is faster and the resulting linear system is more sparse. This property could be an advantage when iterative solution methods are used.

\section{BIBLIOGRAPHY}

1. I. Babuška, The finite element method with Lagrangian multipliers, Numer. Math. 20 (1973), 179-192.

2. I. Babuška and A. Aziz, Survey lectures on the mathematical foundations of the finite element method, in The Mathematical Foundations of the Finite Element Method with Application to Partial Differential Equations (A. Aziz, ed.), Academic Press, New York, 1973, pp. 5359.

3. I. Babuška, J. Osborn, and J. Pitkäranta, Analysis of mixed methods using mesh dependent norms, Math. Comp. 35 (1980), 1039-1062.

4. M. Bercovier and O. Pironneau, Error estimates for finite element solution of the Stokes problem in the primitive variables, Numer. Math. 33 (1979), 211-224.

5. J. Boland and R. Nicolaides, Stability of finite elements under divergence constraints, SIAM J. Numer. Anal. 20 (1983), 722-731.

6. F. Brezzi, On the existence, uniqueness and approximation of saddle-point problems arising from Lagrange multipliers, RAIRO Sér. Rouge 8 (1974), 129-151.

7. F. Brezzi and R. S. Falk, Stability of a higher order Hood-Taylor method, SIAM J. Numer. Anal. (to appear).

8. P. G. Ciarlet, The finite element method for elliptic problems, North-Holland, 1978.

9. P. Clément, Approximation by finite elements using local regularization, RAIRO Sér. Rouge 8 (1975), 77-84.

10. M. Fortin, Old and new finite elements for incompressible flows, Internat. J. Numer. Methods Fluids 1 (1981), 347-364.

11. V. Girault, and P. A. Raviart, Finite element methods for Navier-Stokes equations. Theory and algorithms, Springer, 1986.

12. J. Pitkäranta and R. Stenberg, Analysis of some mixed finite element methods for plane elasticity equations, Math. Comp. 41 (1983), 399-423.

13. R. Scott and M. Vogelius, Norm estimates for a maximal right inverse of the divergence operator in spaces of piecewise polynomials, RAIRO $\mathrm{M}^{2} \mathrm{AN} 19$ (1985), 111-143.

14. R. Stenberg, Analysis of mixed finite element methods for the Stokes problem: A unified approach, Math. Comp. 42 (1984), 9-23.

15. __, On some three-dimensional finite elements for incompressible media, Comput. Methods Appl. Mech. Engrg. 63 (1987), 261-269.

16. R. Verfürth, Error estimates for a mixed finite element approximation of the Stokes equations RAIRO Anal. Numér. 18 (1984), 175-182.

INRIA, Rocquencourt, B.P. 105, 78153 Le Chesnay Cedex, France

Faculty of Mechanical Engineering, Helsinki University of Technology, 02150 Espoo, FinLAND (Current address) 